\title{
Heart Sound Classification from Wavelet Decomposed Signal using Morphological and Statistical Features
}

\author{
Tamanna TK Munia ${ }^{1}$, Kouhyar Tavakolian ${ }^{1,3}$, Ajay K Verma ${ }^{1}$, Vahid Zakeri ${ }^{4}$, \\ Farzad Khosrow-Khavar ${ }^{3}$, Reza Fazel-Rezai ${ }^{1}$, Alireza Akhbardeh ${ }^{2}$ \\ ${ }^{1}$ University of North Dakota, Grand Forks, USA , ${ }^{2}$ Johns Hopkins University, Baltimore,USA, ${ }^{3}$ Simon
Fraser University, Burnaby, Canada, ${ }^{4}$ University of British Columbia, Vancouver, Canada
}

\begin{abstract}
Automatic classification of heart sound recordings is one of the widely known challenges for over 50 years. The fundamental objective of this study is to evaluate a large database of heart sounds collected from a variety of clinical and non-clinical surroundings and classify them into normal and abnormal categories.

Daubechis-2 wavelet transform was applied to each phonocardiogram (PCG) recording after segmenting each cardiac cycle into four windows containing first heart sound S1-Systole-Second heart sound (S2)-Diastole states of a heart cycle. Morphological, statistical and time features were extracted from each cardiac states window. Heart sound classification into normal and abnormal was based on the SVM with Gaussian kernel function. The algorithm was trained by the recordings from all available training data sets (training set A to F). The performance of the proposed prototype was evaluated by five-fold cross-validation on the available training dataset as well as on the hidden test set by PhysioNet. Overall classification accuracies of $82 \%$ during Phase I submissions and $77 \%$ during Phase II submissions were achieved of the challenge. The final score on the blind test set was $74.65 \%$. Based on the current result, the proposed prototype could be a potential solution for a robust and automatic classification technique of normal and abnormal heart sound recordings.
\end{abstract}

\section{Introduction}

Cardiovascular diseases are one of the leading cause of death worldwide. According to World Health Organization's 2015 report, nearly 17.5 million people died from different types of cardiovascular disease in 2012, which represents 31\% of global deaths [1]. Physical examination is one of the first steps to assess the cardiovascular system in clinical practice. An essential part of this physical examination is the heart auscultation, i.e., the act of listening sounds produced by the heart, lungs and blood using a stethoscope to provide information about the valve functioning, rate and rhythm, and anatomical defects of the heart. Auscultation has become official diagnostic method after the invention of the stethoscope in 1816 by Laennec [2]. Thus, by providing critical initial clues in disease assessment, heart sounds serve as a guide for further diagnostic examination and thus play a vital role in the early detection of cardiovascular diseases.

A cardiac cycle is composed of atrial and ventricular contractions produced by the electrical activity generated by the heart. Due to these contractions, the blood flows through different parts of the heart and around the body. The heart valves opening and closure are related with accelerations and decelerations of blood, which eventually give rise to vibrations of the whole cardiac structure (the heart sounds and murmurs) [3]. These vibrations are audible to the chest wall and carry critical information pertaining to the health of the heart. These heart sounds are graphically represented by a signal called phonocardiogram (PCG).

Normally a PCG signal has two major audible sounds (first (S1) and second (S2) heart sounds) which are called fundamental heart sounds. Some other uncommon audible sounds include the third heart sound (S3), the fourth heart sound (S4), systolic ejection click (EC), mid-systolic click (MC), and diastolic sound or opening snap (OS), heart murmurs may occasionally appear due to ageing, disease or mechanical activity of heart such as highvelocity flow of blood [5].

For automatic analysis of heart sound, the first step is to accurately segment the first and the second heart sound for localizing the systolic and diastolic region, so that the pathological situation of this regions can be reliably classified. Analysis of PCG signals, especially the automatic segmentation, and classification has been widely studied for past few decades. Tamer et al. proposed a wavelet-based segmentation and artificial neural network based classification [6]. Guy et al. proposed a clustering based approach in [7] while Chrysa et al. used a segmentation and feature extraction approach 
using ensemble empirical mode decomposition and kurtosis features [8]. Wang et al. and Saracoglu et al. employed Hidden Markov models (HMM) for PCG recordings classification $[9,10]$. Despite the availability of literature, there is still paucity on the sensitivity of the study due to lack of a standardized and high-quality database of heart sound recordings. As a result, the current study intends to develop a robust heart sound classification algorithm from the large public database provided by PhysioNet [11]. Overall, 3,240 heart sound recordings, collected from different locations of the body from both healthy and unhealthy subjects were provided by PhysioNet, as a training dataset for 2016 Challenge. Individual cardiac cycles were extracted from all recordings by using Hidden Semi-Markov Model (HSMM)-based segmentation model. Finally an SVMbased classification methodology was developed by extracting morphological, time and statistical features from segmented heart sounds of wavelet decomposed signal.

\section{Materials and architecture}

\subsection{Database}

The database used in our study was an extensive collection of heart sound recordings obtained from different real-world clinical and non-clinical environments, recorded from both healthy and pathological subjects, and was provided by Physionet/CinC Challenge 2016. The details of the database and data collection environment are provided in [12].

The whole dataset was divided into two parts: training and testing sets. The training set was made available for the challenge participants while test set was kept hidden for scoring purpose. The training set consisted of six databases (A through F) containing a total of 3,240 heart sound recordings, durable from 5 seconds to 120 seconds. All recordings were resampled at $2000 \mathrm{~Hz}$ [11]. The recordings were collected from different locations on the body. In both training and test sets, heart sound recordings were divided into two types: normal and abnormal heart sound recordings.

\subsection{System architecture}

The proposed prototype for automatically classifying a PCG signal into a normal or abnormal was developed through five stages: of cardiac cycle segmentation, wavelet decomposition, further segmentation into four windows (S1-Systole-S2-Diastole), morphological, time and statistical features collection from each window and finally the classification stage to categorize the PCG signal as normal or abnormal heart sound. A detail description of each stage is as follows.

\section{Methodology}

\subsection{Cardiac cycle segmentation}

The first step of automatic heart sounds classification prototype was to segment a PCG signal to localize S1, Systole, S2 and diastole stages of each cardiac cycle. For a segmentation purpose, we have followed the HSMM based algorithm developed by Springer et al. [13]. As shown in Figure 1, the fundamental states of a cardiac cycle S1, Systole, S2 and diastole are clearly distinguishable for a normal heart sound whereas the abnormal heart sound shows no clear visibility for these cardiac states.
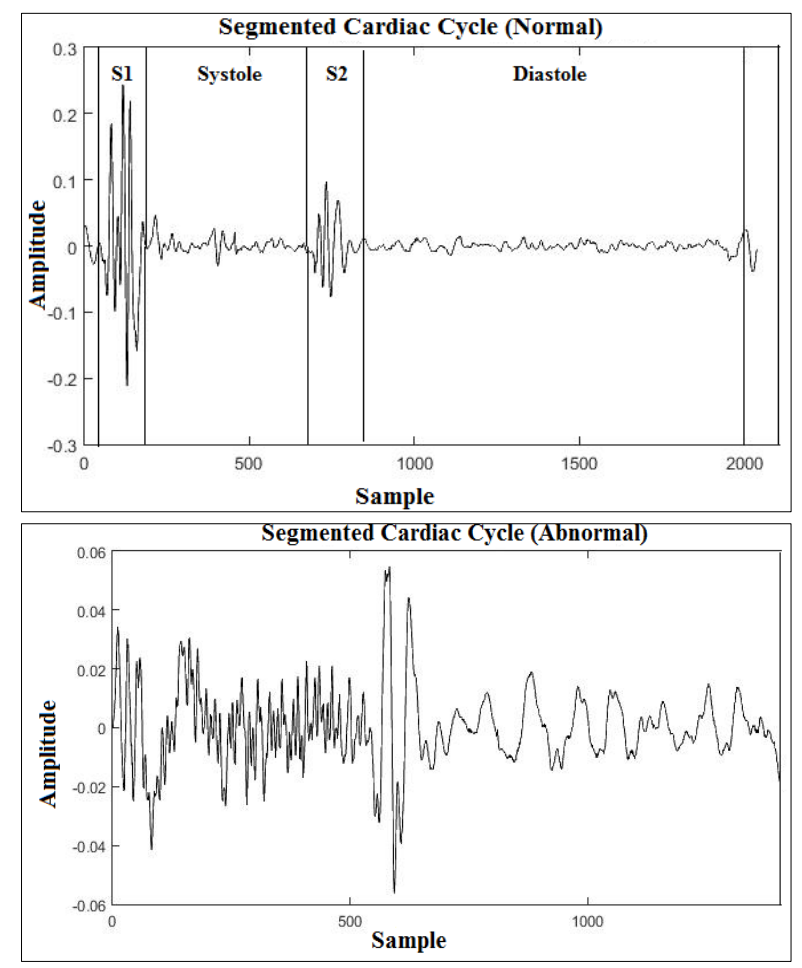

Figure. 1. Segmented cardiac cycle from PCG signal. (a) shows the cardiac cycle segmented from a normal PCG signal showing heart sound S1-Systole-S2-Diastole, while (b) shows the cardiac cycle segmented from an abnormal PCG signal.

\subsection{Wavelet decomposition}

Application of Fourier transform is limited to stationary signals, since PCG signals are non-stationary in nature; we have applied discrete wavelet transform in this stage for sub-band analysis. Wavelet coefficients (approximate and detail) were determined using Daubechies -2 wavelets for each cardiac cycle [14]. For each cardiac cycle, only the detail coefficients at a second decomposition level were used for further processing; this was based on the prior finding in the literature [6] that the detail coefficients at second decomposition level have the 




Figure 2. Four segmented window from a cardiac cycle. Window 1 contains the first heart sound S1, window 2 contains the Systole, window 3 contains the second heart sound and window 4 contains the diastole.

distinguished features, which facilitate differentiating normal and abnormal PCG signals.

\subsection{Segmentation into four window}

In this stage, the signals generated from the detail coefficients of each cardiac cycle were further segmented into four windows such that the window 1 contains the first heart sound S1, window 2 contains the systole stage, window 3 contains the second heart sound and window 4 contains the diastole part of a single cardiac cycle. This segmentation process was performed to extract meaningful features from each stage of a cardiac cycle which can accurately differentiate normal and abnormal PCG signals. Figure 2 shows the four distinct events of a cardiac cycle, namely, S1, systole, S2, and diastole.

\subsection{Feature collection}

The fourth stage was feature collection from each segmented window of a PCG signal. The following features were collected from each signal.

1) Morphological Features: Morphological features extracted from each window were the mean and the standard deviation of a power spectral density of each window ( 2 features per event, total of 8 features), the average dominant frequency of each window (4 features) and, the mean and the standard deviation of linear prediction filter coefficients of each window (8 Features). Thus, a total of 20 morphological features were extracted from a PCG signal.

2) Statistical Features: 16 statistical features were extracted by calculating the mean and standard deviation of skewness (8 features) and kurtosis (8 features) from each window of a cardiac cycle.

3) Time Features: Total 6 time features extracted from each PCG signals, these were the length of S1, systole, S2 and diastole in second (4 features), and ratio of their interval (2 features).

So a total 42 features were extracted from each PCG signal and a feature matrix were generated for the classification purpose in the next step.

\subsection{Classification}

The final stage of the proposed system was to classify the PCG signals into normal and abnormal signals based on the features collected in the previous section. We have used SVM classifier, which is the state-of-art machine learning classification method. The performance of SVM classification was tested for five different kernel functions, i.e., linear, the polynomial of order one, two, and three and the Gaussian. Finally, the Gaussian kernel was selected based on its superior performance in comparison to other kernels.

\section{Results}

The performance of the proposed prototype was evaluated in two different ways. The first way was to calculate the performance measures (accuracy, sensitivity, and specificity) by cross validation method on the six different training datasets available for the challenge participants. The accuracy was calculated using 5 fold cross validation. Table 1 shows the cross-validation results for sensitivity, specificity and accuracy of all available training sets.

Another way of evaluating the performance of the proposed algorithm was to submit the Phase I and Phase II entry for PhysioNet/CinC Challenge 2016, where, the entry was assessed and scored using a hidden test set,

Table 1. Five Fold Cross Validation Results on Training Data Set A to F

\begin{tabular}{|l|cccccccc|}
\hline Dataset & \# Patients & \# recordings & \# Abnormal & \# Normal & \# Unsure & Sensitivity & Specificity & Accuracy \\
\hline Training Set A & 121 & 409 & 276 & 116 & 17 & 0.83 & 0.38 & 0.61 \\
Training Set B & 106 & 490 & 73 & 295 & 122 & 0.36 & 0.88 & 0.62 \\
Training Set C & 31 & 31 & 20 & 7 & 4 & 1.00 & 0.86 & 0.93 \\
Training Set D & 38 & 55 & 26 & 26 & 3 & 0.71 & 0.70 & 0.71 \\
Training Set E & 356 & 2054 & 146 & 1781 & 127 & 0.66 & 0.99 & 0.83 \\
Training Set F & 112 & 114 & 31 & 78 & 5 & 0.38 & 0.89 & 0.64 \\
\hline
\end{tabular}


Phase I entry was evaluated on $20 \%$ of whole test data. The phase II entry was evaluated on a larger portion of the balanced subset from the hidden test set. The final score was based on the whole blind test set. Table 2 shows the phase I, phase II and final entry results for the proposed prototype.

\section{Discussion}

The results shown in Table 1 and 2 indicate that our proposed classification prototype performed better for the training set $\mathrm{C}$ and $\mathrm{E}$ with cross validation than the hidden test set on phase I and phase II entry. The accuracy actually mostly depended on the condition of the dataset if it is balanced or unbalanced. The specificity actually reduced during phase I entry, which potentially could be due to an unequal number of normal and abnormal data on the randomly chosen test data during Phase I entry. During official entry, the balanced entry resulted in higher accuracy than the unbalanced entry.

Table 2. Entry results on Test Set

\begin{tabular}{|c|c|c|c|}
\hline Entry & Sensitivity & Specificity & Accuracy \\
\hline Phase I & 0.76 & 0.88 & 0.82 \\
\hline Phase II & 0.70 & 0.83 & 0.77 \\
\hline Final Score & 0.644 & 0.849 & $\mathbf{0 . 7 4 6}$ \\
\hline
\end{tabular}

The best accuracy reported using this database was 86\% where Potes et al. proposed an Adaboost classifier to distinguish time-frequency based features along with CNN learner based feature collected from segmented heart sounds [15]. Our overall cross-validation accuracy on the whole training set was $81.8 \%$ and on hidden test set was $74.6 \%$. Their hybrid features aggregation method combining the hand crafted and automatic CNN learner based features proved to distinguish the abnormal heart sound efficiently. The wavelet based features collected from each cardiac states in our study is also promising to classify the normal and abnormal heart sound, but further improvement is required to increase the algorithms performance.

\section{Conclusion}

In present study we have explored (i) a methodology for segmenting wavelet decomposed signal in order to extract features from vital cardiac events, namely, S1, systole, S2, and diastole stages of each PCG cardiac cycle, (ii) a set of robust and meaningful features by combining morphological, statistical and time features which has potential to accurately classify heart sounds obtained under practical conditions, and (iii) a reliability of SVM-based classifier with Gaussian kernel function was validated which performed better than other classifiers both on the training dataset and hidden test set to classify the normal and abnormal PCG signals.
The future steps for this study could be explorations into the extraction of new and novel features to further increase the accuracy and to apply a feature selection methodology like divergence analysis in order to select best features, which will eventually improve the accuracy.

\section{References}

[1] WHO 2015 World statistics on cardiovascular disease (http://www.who.int/mediacentre/factsheets/fs317/en/

[2] Laennec, Hyacinthe RT, \& Forbes J, (1838). "A Treatise on the Diseases of the Chest and on Mediate Auscultation".

[3] Leatham, A. Auscultation of the heart and phonocardiograph. Churchill Livingstone: 1975.

[4] Obaidat MS "Phonocardiogram signal analysis: techniques and performance comparison." Journal of Medical Engi. \& Technology 17, no. 6 (1993): 221-227.

[5] Dormat A, and J. Bourneuf D. "Nouveau Larousse Médical." Paris, Librairie Larousse (1981).

[6] Ölmez T, and Zümray D. "Classification of heart sounds using an artificial neural network". Pattern Recognition Letters 24, no. 1 (2003): 617-629.

[7] Guy A, Gavriely N, and Intrator N. "Cluster analysis and classification of heart sound." Biomedical Signal Processing and Control 4, no. 1 (2009): 26-36.

[8] Papadaniil, Chrysa D., and Leontios J. Hadjileontiadis. "Efficient heart sound segmentation and extraction using ensemble empirical mode decomposition and kurtosis features." Biomedical and Health Informatics, IEEE Journal of 18, no. 4 (2014): 1138-1152.

[9] Wang JP.; Lim CS, Chauhan S, Foo JY, Anantharaman V, Phonocardiographic signal analysis method using a modified hidden Markov model. Ann Biomed Eng 2007, 35, 367-374.

[10] Saracoglu R. Hidden Markov model-based classification of heart valve disease with PCA for dimension reduction. Eng Appl Artif Intell 2012, 25, 1523-1528.

[11] Goldberger AL, Amaral LAN,Glass L, Hausdorff JM, Ivanov PCh,Mark RG,Mietus JE,Moody GB, Peng CK, Stanley HE. "PhysioBank, PhysioToolkit, and PhysioNet: Components of a New Research Resource for Complex Physiologic Signals," Circulation 101(23):e215-e220 [Circulation Electronic Pages; 2000 (June 13).

[12] Liu et al., "An Open Access Database for the Evaluation of Heart Sound Algorithms", Physiol. Meas. Vol 37, number11, (In Press) 2016.

[13] Springer DB, Tarassenko L, Clifford GD. Logistic Regression-HSMM-based Heart Sound Segmentation. IEEE Transactions on Biomedical Eng., In press; 2015.

[14] Ingrid D., and Bates BJ. "Ten lectures on wavelets." The Journal of the Acoustical Society of America 93, no. 3 (1993): 1671-1671.

[15] Potes C, Parvaneh S, Rahman A, Conroy B. Classifier ensemble for detection of abnormal heart sounds. Computing in Cardiology 2016;43.

Address for correspondence.

Kouhyar Tavakolian

Full postal address. Upson Hall II Room 160, 243 Centennial Drive Stop 7165, Grand Forks, ND 58202, USA

E-mail address: kouhyar.tavakolian@engr.und.edu 